Original paper

\title{
A comparison among different techniques for human ERG signals processing and classification
}

\author{
R. Barraco ${ }^{\text {a }}$, D. Persano Adorno ${ }^{\text {a, } *}$, M. Brai ${ }^{\text {a }}$, L. Tranchina ${ }^{\mathrm{b}}$ \\ a Dipartimento di Fisica e Chimica, Università di Palermo and CNISM, Viale delle Scienze, Ed. 18, I-90128 Palermo, Italy \\ ${ }^{\mathrm{b}}$ Laboratorio di Fisica e Tecnologie Relative - UniNetLab, Università di Palermo, Viale delle Scienze, Ed. 18, I-90128 Palermo, Italy
}

\section{A R T I C L E I N F O}

\section{Article history:}

Received 19 August 2012

Received in revised form

23 January 2013

Accepted 19 March 2013

Available online 13 April 2013

\section{Keywords:}

ERG signals

Wavelet analysis

Principal component analysis

Retinal pathologies

\begin{abstract}
A B S T R A C T
Feature detection in biomedical signals is crucial for deepening our knowledge about the involved physiological processes. To achieve this aim, many analytic approaches can be applied but only few are able to deal with signals whose time dependent features provide useful clinical information. Among the biomedical signals, the electroretinogram (ERG), that records the retinal response to a light flash, can improve our comprehension of the complex photoreceptoral activities.

The present study is focused on the analysis of the early response of the photoreceptoral human system, known as a-wave ERG-component. This wave reflects the functional integrity of the photoreceptors, rods and cones, whose activation dynamics are not yet completely understood. Moreover, since in incipient photoreceptoral pathologies eventual anomalies in $a$-wave are not always detectable with a "naked eye" analysis of the traces, the possibility to discriminate pathologic from healthy traces, by means of appropriate analytical techniques, could help in clinical diagnosis.

In the present paper, we discuss and compare the efficiency of various techniques of signal processing, such as Fourier analysis (FA), Principal Component Analysis (PCA), Wavelet Analysis (WA) in recognising pathological traces from the healthy ones. The investigated retinal pathologies are Achromatopsia, a cone disease and Congenital Stationary Night Blindness, affecting the photoreceptoral signal transmission. Our findings prove that both PCA and FA of conventional ERGs, don't add clinical information useful for the diagnosis of ocular pathologies, whereas the use of a more sophisticated analysis, based on the wavelet transform, provides a powerful tool for routine clinical examinations of patients.
\end{abstract}

( 2013 Associazione Italiana di Fisica Medica. Published by Elsevier Ltd. All rights reserved.

\section{Introduction}

The purpose of biomedical data processing is to extract from signal measurements, by using mathematical methods, relevant information as characteristic features, in order to enable an improved medical diagnosis. Biomedical signals are extremely difficult to process and interpret since they are the combination of intricate physiological processes that reflect the activities of complex systems, whose composition can be time dependent. Therefore, the use of analytical techniques, able to extract hidden features in signals of this type, is becoming an important tool in clinical context; in fact, after the identification of characteristics of signals considered clinically normal, the recognition of deviations by them could be indicative of a disease/disorder condition. Nevertheless, the complexity of phenomena involved in the generation processes makes this task not always immediate. So, the

\footnotetext{
* Corresponding author. Tel.: +39 (0)9123899086.

E-mail address: dominique.persanoadorno@unipa.it (D. Persano Adorno).
}

individuation of an appropriate analytical approach for revealing signal peculiarities, can become an advantageous goal for detecting pathological conditions.

Generally speaking, any signal transduced from a biological or medical source can be considered a biosignal. It is, then, a collective electrical signal, associated to a physiological process, that represents a physical variable of interest. It is, normally, function of time and can be described in terms of its amplitude, frequency and shape and can exhibit non stationary characteristics. The study of physiological processes through the analysis of related biosignals, has attracted the interest of many researchers who have explored and studied a variety of signals of this type, including the best-known electrocardiogram (ECG), the electroencephalogram (EEG), the electromyogram (EMG), polysomnography, etc [1-6].

This paper deals with a biomedical signal interesting for its intrinsic characteristics and diagnostic potential: the electroretinogram (ERG). It holds a place of relevant importance in ocular physiology since it is a powerful test in the evaluation of the functional status of the retina. The ERG records the action currents produced in the retina in response to light stimuli of various type 
and consists of a sequence of components ( $a-, b-, c$-, $d$-wave ones, oscillatory potentials, etc.), originated in different retinal layers. Understanding the specific features (onset, time delay, amplitude, line shape) of the ERG components and their relationships represents the principal aim of past and present research in the field of ocular electrophysiology [7-18] because this information can be useful in discovering the nature of certain retinal disorders and in early diagnosing retinal diseases [19-21].

Different techniques can be utilised in ERG signal processing, including the principal component analysis (PCA), the consolidated Fourier analysis (FA), the more recent wavelet analysis (WA), etc. All, within their capabilities, can be a useful aid in clinical diagnosis, especially in cases in which the physicians need additional clinical investigations. In particular, we have analysed data from both healthy subjects and patients affected by two retinal pathologies, congenital achromatopsia (ACHM) and X-linked congenital stationary night blindness (CSNB).

The goal of this study is to design and develop a new method for automatic recognition of the electroretinogram records in order to speed up and objectify the identification of ocular pathologies. In fact, till now, to the best of our knowledge, the identification is always based on visual analysis of the records, eventually integrated with other clinical exams.

\section{Materials and methods}

\section{ERG: physiological background and signal acquisition}

The ERG represents the global response of the retina to a flash stimulation and reflects the functionality of photoreceptors and inner nuclear layers of the retina [7,9,11,22-25]. The initial ERG signal shape up to $\sim 30 \mathrm{~ms}$ (a negative potential marked by one or two dips) is known as $a$-wave and, in normal subjects, it is generally ascribed to the contribution of cone and rod activities [26,27].

The $a$-wave is followed by the positive $b$-wave, arising from the activation of deeper retinal layers and by other waves. The amplitude and temporal pattern of ERG $a$-wave component, depends on: i) the functional integrity of the retina; ii) the intensity of test flash reaching the retina and, iii) the ambient illumination. A photoreceptoral pathology can substantially modify the $a$-wave or only slightly affect it, in manner not always detectable upon visual inspection and evaluation. The pathologies here investigated, ACHM and CSNB, represent two different examples of these possibilities.

ACHM is a hereditary disease due to a lack of cone vision. There are two forms in which ACHM can subsist: incomplete and complete. In general, the eyes affected by ACHM, lacking normal cone vision, are not able to normally adapt to high levels of illumination; ACHM patients are completely colour-blind, or nearly so, and have very poor visual acuity. Because there are many variations in the severity of the pathology among individual achromates, it is not simple to identify its different forms.

CSNB is a rare inherited non-progressive retinal disorder, consequence of the impaired transmission between the photoreceptors and the bipolar cells further down the visual pathway. CSNB patients have a reduced sharpness of vision, night blindness and have difficulty in adapting to low light situations due to impaired photoreceptor transmission. It has two forms, complete, also known as type-1 (CSNB1), and incomplete, also known as type-2 (CSNB2), depending on severity. In the complete form (CSNB1), there is no measurable rod cell response to light, whereas this response is measurable in the incomplete form.

For purpose of the study, the investigated ERG sample consists of normal ERG waveforms and ERG recordings typical of the two retinal diseases. Figure 1 displays the set of the $a$-waves, object of the present investigation, acquired at the Department of Sensory
Neurophysiology (Sect. Ophthalmology) of the Palermo University. The traces shown in the panels are representative of 24 different records, belonging to 12 patients whose demographic characteristics are reported in Table 1 . The ERG signals of both eyes of each subject were recorded and analysed. The subjects not affected by ocular diseases have visual acuity of 20/20 (range \pm 2 ) and show negligible differences between the two eyes.

The ERGs were recorded by using instrumentation manufactured by Biomedical Mangoni, following routine methods by means of Henke's corneal electrodes [28,29]. The electrodes were frontal, with the ground electrode on the forehead. Electrode impedance was kept under $5 \mathrm{k} \Omega$. Stimuli were stroboscopic white flashes presented in a Ganzfeld integrating sphere of $40 \mathrm{~cm}$ diameter. The repetition interval of the stimulus was $15 \mathrm{~s}$ and its duration was $50 \mu \mathrm{s}$. There is dissimilar information about the best choice of the band pass filter in ERG acquisition. Robson and Frishman carried out an extensive analysis using filters with different band widths [30]. They concluded that the ERG waveform, in any case, does not contain frequency components greater than $500 \mathrm{~Hz}$, and some distortion occurs when narrow band pass filters are used. Following these achievements and taking into account that an ideal choice does not exist, the amplifier band pass has been setted in the range $1-250 \mathrm{~Hz}$, with low pass slope $12 \mathrm{~dB} /$ octave (Butterworth second order) and high pass slope $6 \mathrm{~dB} /$ octave (first order). The cut off frequency corresponds to the point $-3 \mathrm{~dB}$. The input impedance of the preamplifier was $10 \mathrm{M} \Omega$. The order of the filter and amplifier requirements were in line with the ISCEV standards [29]. The standard luminance, denoted by $I_{0}$, was $1.7 \mathrm{~cd} \mathrm{~m}^{-2}$. Here, we investigate the traces recorded with the $\log (I /$ $\left.I_{0}\right)=1.5$ luminance value. All patients were subjected to maximal papillary dilatation $(\geq 7 \mathrm{~mm})$ with Nethyl-( $\alpha$-picolyl) tropicamide $1 \%$, and the cornea was anesthetized using oxybuprocaine hydrochloride $4 \%$. They were, then, dark-adapted (scotopic ERG) for 30 min, in accordance with the standards for clinical electroretinography $[28,29]$. Each record lasted $500 \mathrm{~ms}$ with sampling time $\delta t=0.1953 \mathrm{~ms}$. Each trace was obtained by averaging at least 3 responses. Data were sampled with a Nyquist frequency of $2560 \mathrm{~Hz}$ and stored as ASCII files for subsequent retrieval and analysis.

The traces shown in Fig. 1, all recorded with the same luminance value, are not immediately distinguishable in relation to their belonging either to healthy ERGs or to pathological cases. In fact, an expert clinician for their identification and classification, beyond the objective examination of the traces, needs other clinical investigations that could imply a greater amount of time, costs and, eventual patient displacement. Figure 2 shows the classification into groups made by a medical practitioner after the integration of the visual analysis with further clinical ophthalmologic investigations. In particular, panel (a) shows 10 healthy ERGs; panels (b) and (c) display 8 ACHM (incomplete form) and 6 CSNB (type-1) waveforms, respectively. The time interval used in the figures spans over $30 \mathrm{~ms}$. At greater times, an overlap between the tail of the $a$-wave and the beginning of the successive $b$-wave begins to take place.

\section{Methods in ERG signal processing and classification}

Raw biomedical signals contain valuable information that can be extracted by means of advanced analytical methods for detecting characteristic features. The basic approach to signal analysis is then to get proper information from the signal by applying the best suitable method. Different techniques have been introduced for identification of signal features, we here present some that could be used for analysing ERG signal.

\section{Principal component analysis (PCA)}

PCA is a widely used mathematical technique able to discover hidden patterns in confusing data sets and to provide a data 

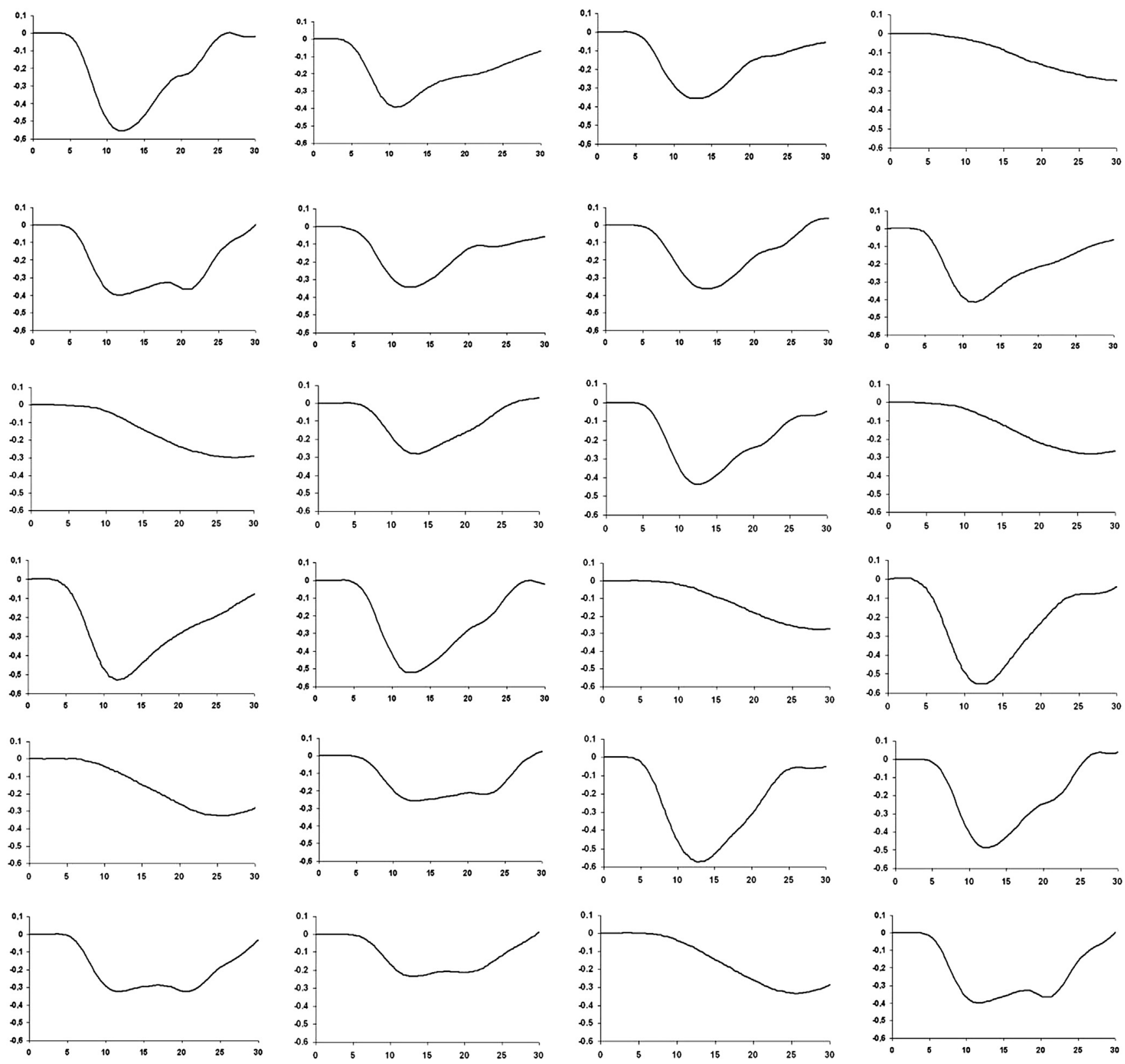

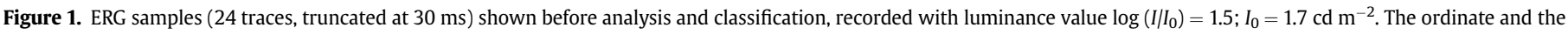
abscissa report the retinal response values $(\mathrm{mV})$ and the time values $(\mathrm{ms})$, respectively.

representation suitable for highlighting the differences between different trials. In fact, the extraction of relevant features from the cluster structure is useful as visualization tool since it provides a low dimensional summary of the data helping to uncover hidden trend in the data, to detect outliers, and perform quality control.

These peculiarities make PCA very versatile and applicable to a variety of tasks: classification and recognition problems [31], image

Table 1

Demographic characteristics of the patient population.

\begin{tabular}{llll}
\hline Diagnosis & Number & Age \pm SD & Gender \\
\hline Normal subjects & 5 & $35 \pm 14$ & $2 \mathrm{M} / 3 \mathrm{~F}$ \\
ACHM & 4 & $41 \pm 19$ & $2 \mathrm{M} / 2 \mathrm{~F}$ \\
CSNB & 3 & $37 \pm 12$ & $1 \mathrm{M} / 2 \mathrm{~F}$ \\
\hline
\end{tabular}

processing [32], signal modelling, spectral estimation, array processing [33] and biosignal analysis [34-36].

Moreover, PCA results particularly valuable in all those cases in which many factors (variables) or data multidimensionality affect the data to be analysed. It is, in fact, useful when one has to deal with data affected by the combination of a large number of variables that could be redundant, i.e. correlated with one another. In this sense, PCA is a statistical technique aimed to condense the information of a large set of correlated variables into a few variables (called principal components), without neglecting the variability characterizing the data set.

This multivariate analysis, hence, can be considered, as a rotation of the coordinate axes so that the new axes are along linear combinations of sets of time points altogether representing a pattern within the signal. This rotation is achieved by maximising the variance within the data along the first axis, maintaining the 

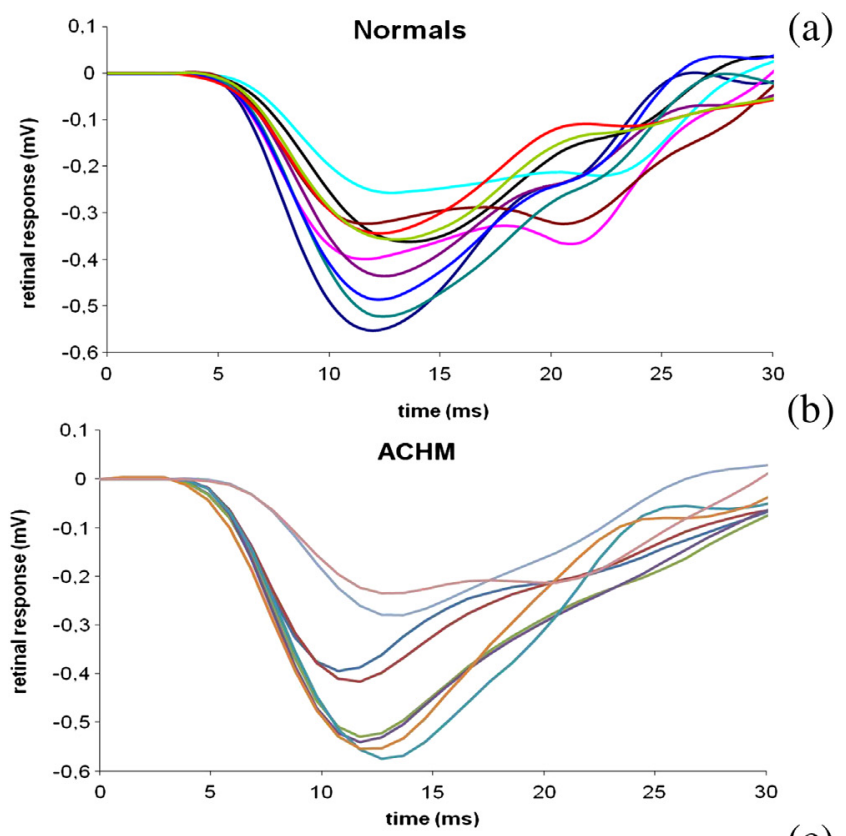

(c)

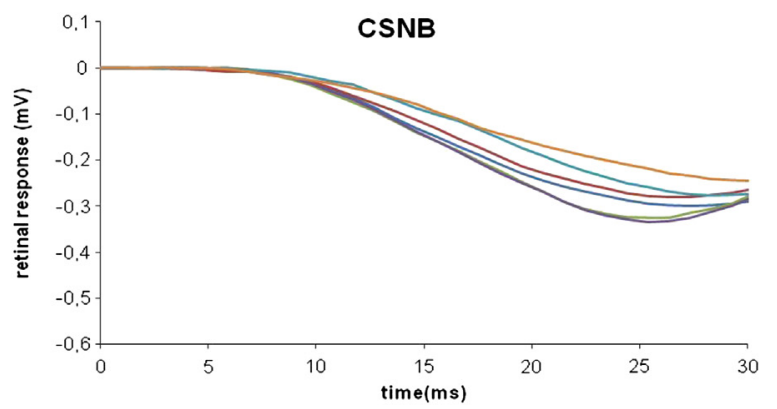

Figure 2. ERG samples (truncated at $30 \mathrm{~ms}$ ) recorded with luminance value log (I) $\left.I_{0}\right)=1.5$. Human healthy, ACHM and CSNB waves are shown in upper, middle and bottom panel, respectively.

orthogonality of the axes. These new variables, called principal components or factors, are then linear combination of the originals and account for most of the variance in the original variables. In particular, the first factor accounts for the greater percentage of this variance, the second factor less and, so on. In other words, in the rotated coordinate system the first axis represents the important features within the data set, whereas the second axis encodes how much the two variables of a data point deviate from their average relation within the whole data set.

In this way, $100 \%$ of the variance is explained by a number of factors equal to the number of analysed variables. The factor loadings are represented by the correlation coefficients between the original variables and derived factors. The principal components are mutually uncorrelated.

In our study PCA is performed by using the Statistics Package for Excel - XLSTAT Addinsoft xlstat 2009 software.

\section{Fourier analysis (FA)}

One of the most common analysis techniques used in signal processing is the spectral analysis, i.e. the decomposition of the signal into its frequency components. Among the mostly used spectral analysis methods, the FA both for historical and analytical reasons, plays an important role, and is considered preliminary to any other method of spectral type. It, in fact, is able to evaluate the frequency components of a signal and their amplitude, providing a broad overview of its features. Nevertheless, the spectral analysis based on the classical Fourier transform (FT) assumes the signal to be stationary, ignoring that it can have a time-varying spectral content. Then, FT is inherently non local, due to the analytical characteristics of the trigonometric functions and, consequently, not suitable to analyse non stationary signals, as biosignals are. Alternative techniques capable to determine the spectral content, localised in time, can be used in dealing with non-stationary signals. The more common used algorithms for time-frequency representations are Short-Time Fourier Transform (STFT), Wigner-Ville transform, Wavelet Transforms, etc. Fourier spectra are, here, represented with bar graphs showing the detected frequencies in the set of analysed traces.

For the Fourier analysis a custom-designed software, based on the use of the fast Fourier transform (FFT) algorithm, as described in the Numerical Recipes, is developed and utilized.

\section{Wavelet analysis (WA)}

Differently from the FA, the WA can be locally performed on the signal, with the aim of extracting time-frequency features characterizing it. Recently, it has been applied to problems covering different areas of both applied mathematics and physics, including engineering, biology and medicine [1-4,37-42]. The wavelet transform (WT) is appropriate to reveal the spectral behaviour of complex and/or non-linear processes, characterized by interactions of varied nature. They are based on group theory and square integrable representations, which allow us to unfold a signal into timefrequency space.

We, here, use the continuous wavelet transform (CWT) that furnishes, in the time-frequency plane, a resolution better than other methods of computing the transforms; so that an accurate identification and partitioning of the characteristic features of the signal are possible. CWT overcomes the problems of the Fourier transform using variable-length windows (smaller windows capture a broader frequency content providing good temporal localization; larger windows capture lower frequency content ensuring good frequency resolution). The kernel functions used in WT $[43,44]$ are derived from a prototype function (mother wavelet) by translation controlled by the scale parameter $\sigma$ (related to the frequency band) and the translation parameter $\tau$ (related to the temporal position of the window).

High values of $\sigma$ allow rough analysis of the signal. Conversely, low values of $\sigma$ can capture the detailed information. The coefficients of CWT can be easily evaluated by means of the equation:

$\mathrm{WT}(\tau, \sigma)=\frac{1}{\sqrt{\sigma}} \int_{-\infty}^{+\infty} x(t) \psi^{*}\left(\frac{t-\tau}{\sigma}\right) \mathrm{d} t$

where $x(t)$ is the signal to be analysed and $\psi$ is the chosen wavelet. The transform tends to assume extreme values when the signal pattern tends to become similar to that of the wavelet. Therefore, the WT may be considered as a measure of similarity between the signal to be analysed and the mother wavelet at that instant. This correlation between the mother wavelet and signal can be explored through the presence of peaks in 3-dimensional plots which display the coefficients of CWT as function of $\sigma$ and $\tau$. A better visualisation of this spectral behaviour results by projecting the coefficients in the $(\sigma, \tau)$ plane. In the latter case, the correlation is connected with the presence of clusters in the corresponding contour plots [45]. As a consequence of the Heisenberg uncertainty relation, the WA gives good time resolution and poor frequency resolution at small scales (high frequencies), whereas it provides good frequency resolution and poor time resolution at large scales (low frequencies). 
Since each wavelet has specific features in time and frequency domains, the use of a particular mother wavelet can emphasise different characteristics of the signal. In our case, the choice of the most suitable mother wavelet has been preceded by a preliminary analysis aimed to select the function that best adheres to the shape of the ERG data. On the basis of previous works, showing the Gaussian as a robust statistical description of the $a$-wave both for healthy and ACHM subjects [26,27], we have chosen the Mexican Hat (MHW), second derivative of a Gaussian function, as appropriate mother wavelet for analysing our waveforms. The MHW is defined by:

$\operatorname{MHW}(t, \tau, \sigma)=\frac{1}{\sqrt{2 \pi}}\left[1-\left(\frac{t-\tau}{\sigma}\right)^{2}\right] \cdot e^{-\frac{1}{2}\left(\frac{t-\tau}{\sigma}\right)^{2}}$

It is continuous, non orthogonal and contains a small number of regular oscillations that build up and decay over the period of time of interest.

Differently from other mother wavelets (Haar, Morlet, Daubechies), whose shapes are not similar to our traces, the MHW gives results in good agreement with the preliminary FA and for normal subjects has provided a good detection and localization of the characteristic features of the signal in regions of stationary activity that correspond either to minima or to maxima $[38,39]$. There exist different methods of displaying the results: we, here, calculate the absolute value of $\operatorname{WT}(\sigma, \tau)$ coefficients and then normalize it to its maximum value for each trace [45-47]:

$\mathrm{WT}_{\mathrm{abs}}(\sigma, \tau)=\frac{\operatorname{abs}(\mathrm{WT}(\sigma, \tau))}{\max [\operatorname{abs}(\mathrm{WT}(\sigma, \tau))]}$

The relevant frequencies are identified through the existence of clusters in the corresponding colour scalograms, that show the correlations among MHW and signal.

The time-frequency spectrum of a trace is shown as a shaded contour plot of $\mathrm{WT}_{\mathrm{abs}}(t, f)$ where the minimum values of the absolute values of the normalised wavelet coefficients are in purple, and the maximum ones in white. The correlation magnitudes are expressed as a pink-scale in terms of a scalogram with relative magnitude normalized to the peak value for each trace (according to Eq. (3)) [45-47].
The electroretinographic records are processed by using the wavelet toolbox of MATLAB.

\section{Results and discussion}

Before dealing with sophisticated analysis, not standard in clinical protocol, we have carried out a simple analysis of both slope and implicit time of our a-wave set, in order to evaluate its capability to discriminate healthy from pathological traces. Here, the implicit time was determined by the intersection of a straight line drawn along the initial $a$-wave downslope with the amplitude baseline, according to Ref. [48]. This preliminary analysis, of course, is successful in classifying the CSNB ERGs, but it provides confusing results on normal and ACHM traces. The results obtained for normal and ACHM traces are, respectively:

$$
\begin{array}{ll}
\text { Healthy ERGs : } & \text { slope }(\mathrm{mV} / \mathrm{ms})=-0.0694 \pm 0.0217 \\
& \text { implicit time }(\mathrm{ms}): 11.532 \pm 1.403
\end{array}
$$

$$
\begin{array}{ll}
\text { ACHM ERGs: } & \text { slope }(\mathrm{mV} / \mathrm{ms})=-0.0763 \pm 0.0213 \\
& \text { implicit time }(\mathrm{ms}): 10.956 \pm 0.758
\end{array}
$$

where we report the mean of both slopes and implicit times, calculated within each sample and the standard deviation. Both sets of parameters show a considerable range of variance as indicated by the great value of standard deviation. In order to have a statistical evaluation of the reliability of this simple technique as classification algorithm, we run the $t$-test with these outcomes, obtaining differences in the mean values of the two groups not great enough to reject the possibility that the differences are due to random sampling variability. Namely, there is not a statistically significant difference between the input groups. Then, in our case, a simple analysis of both slope and implicit time of $a$-waveforms, can't provide enough information clinically-useful, for example, in the recognition of early or subtle ocular diseases.

According to the aim of this work, in the following we discuss the results obtained by using more advanced investigations, such as the three analytical methods introduced above. We, then, compare their capability to differentiate normal from pathological ERGs and to extract valuable information useful in diagnosis, improving the individuation of the development stage of progressive diseases of retinal pathways.

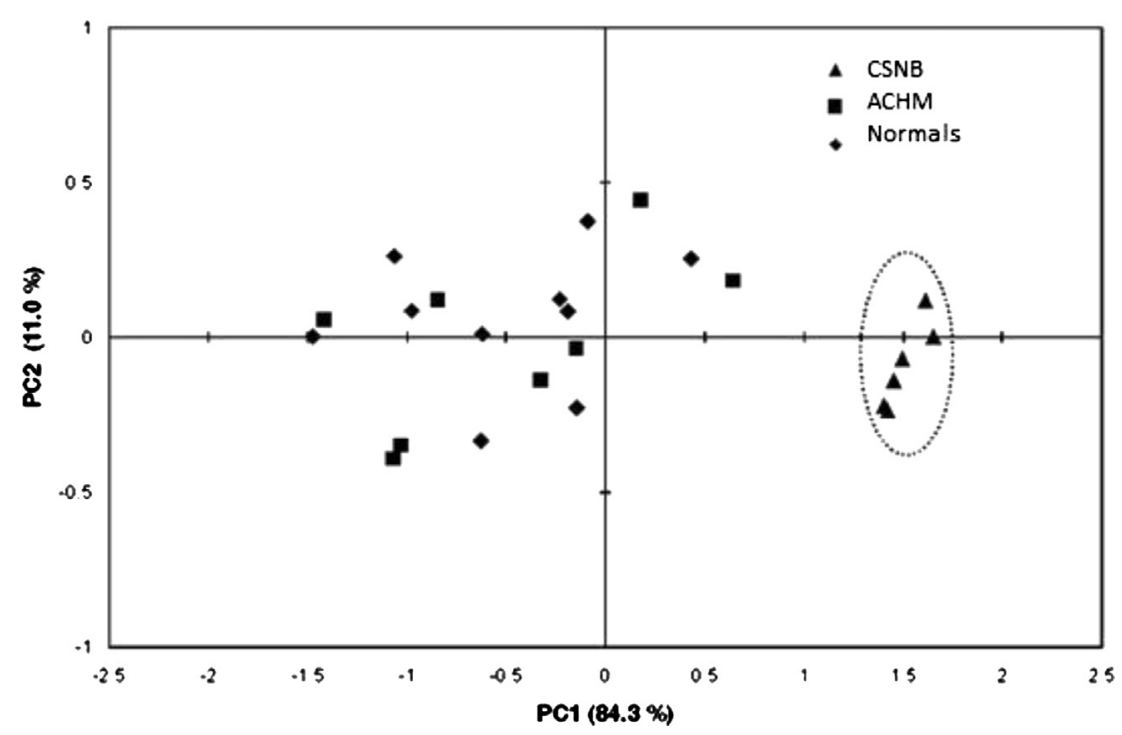

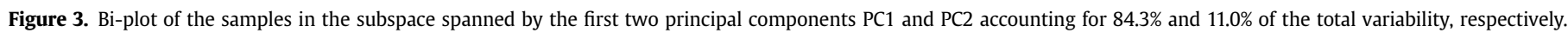




\section{Principal component analysis results}

The PCA has been the first analytical approach used in signal processing with the aim of evaluating its effectiveness in highlighting possible cluster structures within the sample of analysed waves. We explore the possibility to extract features characterising the three types of traces through the peculiarities of the multivariate analysis.

Figure 3 shows a bi-plot of the new observation coordinates in the first and second principal component (PC1 and PC2) extracted by PCA analysis. As one can see these two components, account for the $95.3 \%$ of the overall variance. In particular, PC1 and PC2 account for $84.3 \%$ and $11.0 \%$, respectively.

Unfortunately, PCA turns out not to be quite reliable in the classification of normal and pathological traces. It, in fact, regroups and identifies, by clustering (group in the right side of the graph) only the traces CSNB (triangles), whereas it is not able to clearly separate the ACHM traces (squares) from healthy ones (diamonds). The correlations among the variables (signal intensity at time $t$ ) and the extracted factors PC1 and PC2 are reported in Fig. 4. This figure shows that signal intensities in the 8-19 ms time interval are strongly related to the factor PC1 with correlation coefficients $>0.90$. The position of the CSNB traces flattened along PC1 in the biplot is hence strongly related to their different signal behaviour with respect ACHM and healthy in the 8-19 ms time interval. No significant differences can be highlighted between ACHM and healthy responses.

\section{Spectral analysis results}

\section{Fourier analysis}

An FA aimed to select the range of the frequency components contained in the ERG spectrum was carried out. At first, the analysis was performed over a time interval $(50 \mathrm{~ms})$, greater than that of interest, both to avoid undesirable effects at the edges and to obtain an enough good resolution of FFT. The FFT spectrum of all subjects (healthy, ACHM and CSNB) contains a dominant high intensity component at low frequency $(\sim 20 \mathrm{~Hz})$, that is not further considered since it does not give relevant information. On the other hand, higher frequency components, with coefficients appreciably smaller, are only present in normal and ACHM spectra. Therefore, the FA, as the PCA, is able to isolate CSNB traces.

Figure 5 reports the mean values of the FFT coefficients in the high frequency range $(80-240 \mathrm{~Hz})$, also showing the percentage of subjects in which each frequency component is found, for the set of healthy (top panel) and ACHM (bottom panel) traces. Beyond

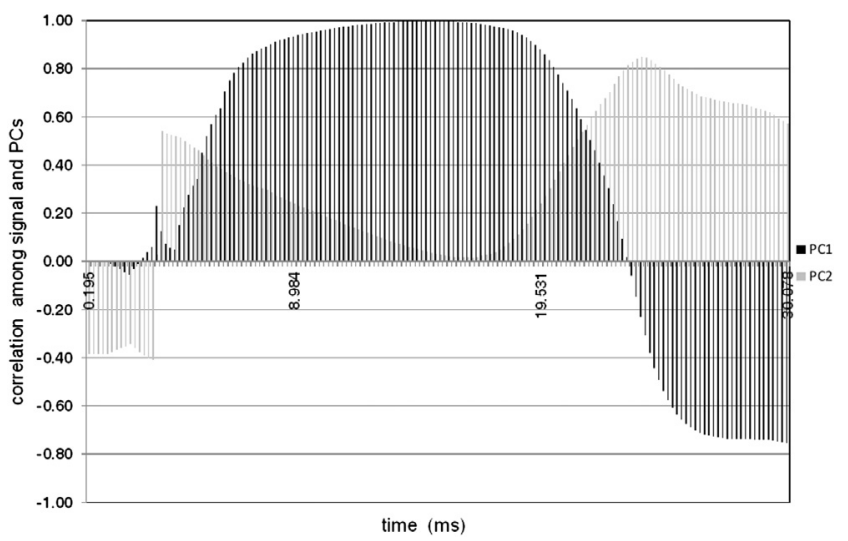

Figure 4. Correlation values (ordinate) among the retinal response intensity at the time $t$ (abscissa) and the extracted factors PC1 (black bars) and PC2 (gray bars).
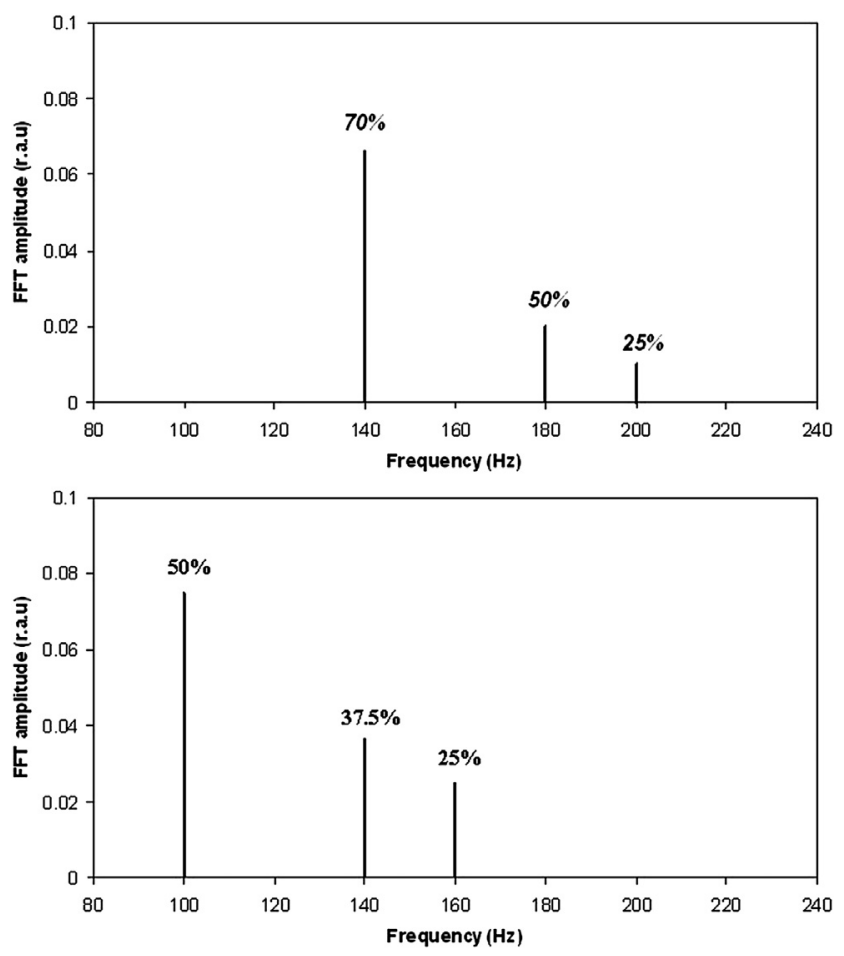

Figure 5. Mean values of the FFT coefficients (normalized to their maximum value) in the high frequency range, showing also the percentage of subjects in which each frequency component is present, calculated for the healthy (upper panel) and the ACHM (bottom panel) traces shown in Fig. 1.

$240 \mathrm{~Hz}$, appreciable components have not been found, according to the acquired evidence that $a$-wave is a low frequency signal. The interpretation of results shown in Fig. 5 is difficult and potentially controversial. In fact, one possible conclusion is that the FA is unable to clearly distinguish between $a$-waves recorded from normal subjects or ACHM patients since it detects, in 37.5\% of ACHM cases, the component frequency of $140 \mathrm{~Hz}$, found also in $70 \%$ of normals. This circumstance could introduce the problem of false positives, making the FA not completely reliable as a tool for the recognition

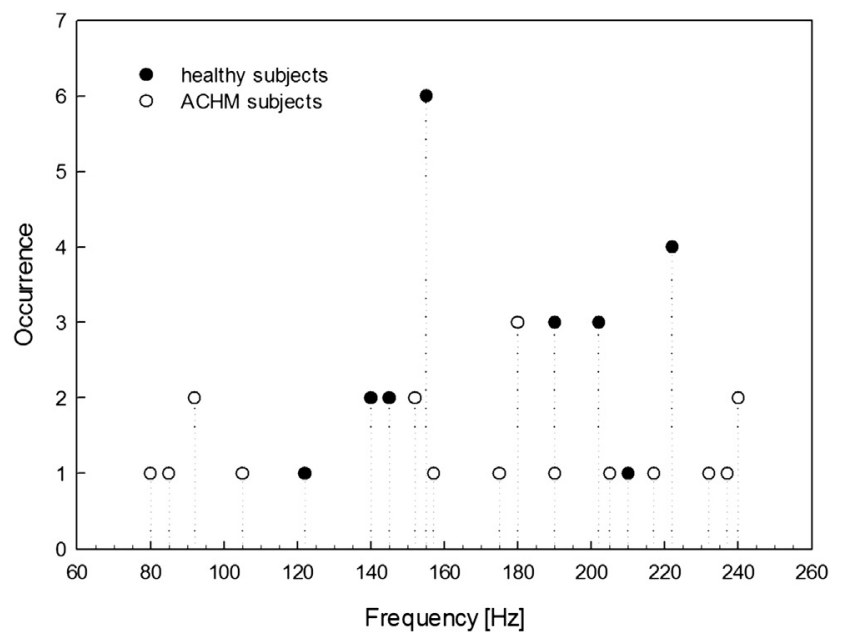

Figure 6. Frequency components for both healthy (full circles) and ACHM (empty circles) traces versus their occurrence, obtained by enlarging the acquisition time up to $500 \mathrm{~ms}$, but investigating only the negative potential up to the cross-over with the time axis and leading to zero the remaining part of ERG signal. 


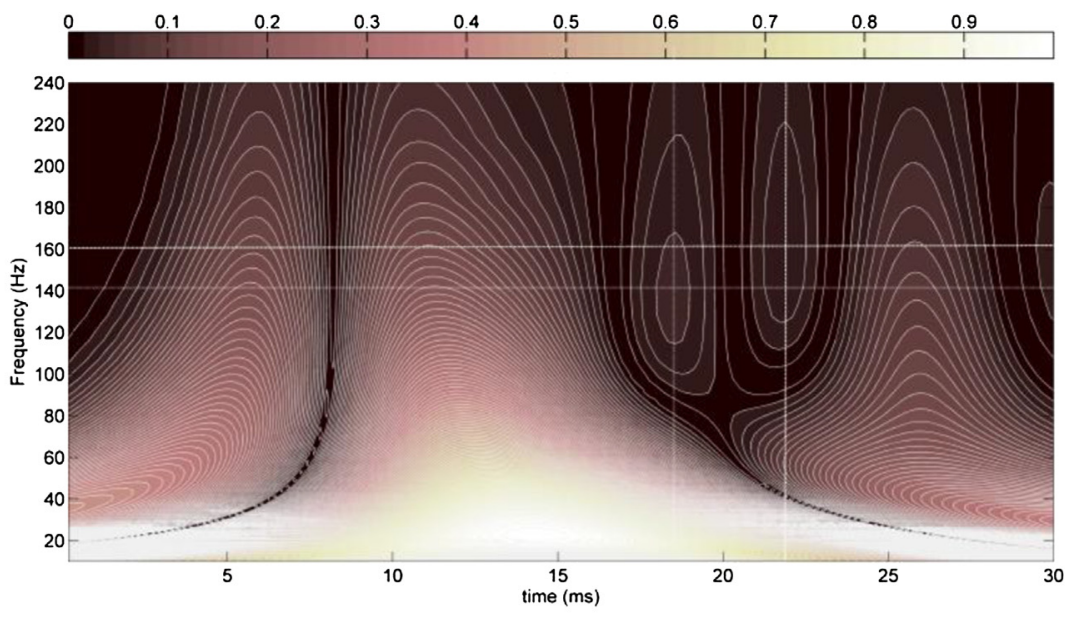

(a)

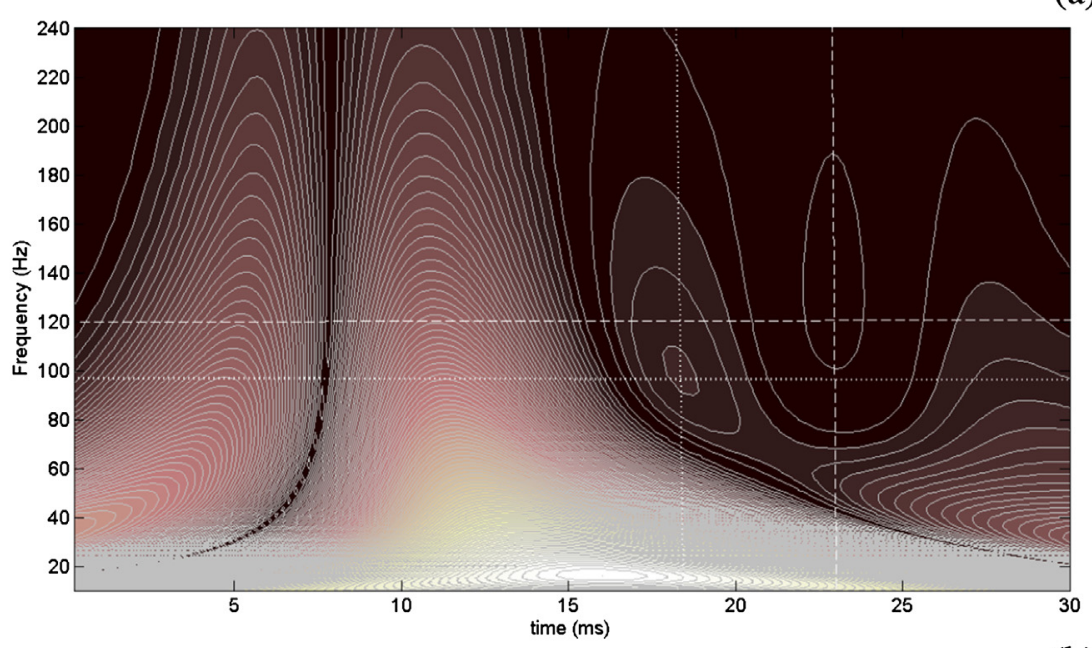

(b)

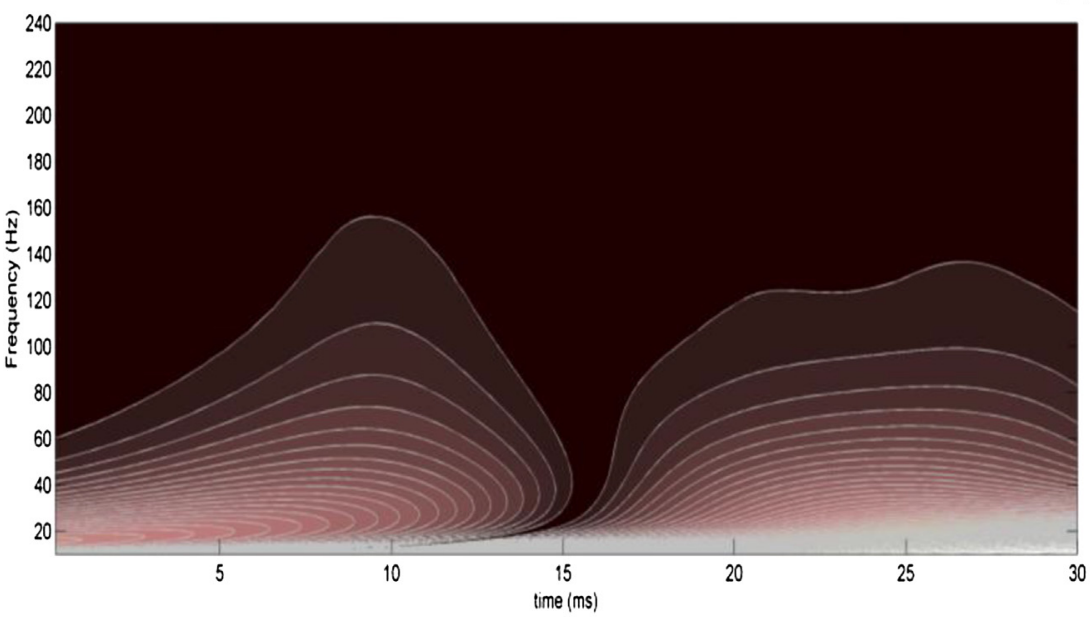

(c)

Figure 7. Spectral behaviour of a trace representative of a subject of the healthy sample (panel a), ACHM (panel b) and CSNB sample (panel c). The panels show the amplitude of the transform in the range from 10 to $240 \mathrm{~Hz}$ across time as a colour scalogram. A scale of colours tending to white denotes the high-energy components, the pink scale denotes the middle energy the low-energy components in the $30 \mathrm{~ms}$ time interval. The straight lines identify the coordinates of the local maxima, $f_{1}$ and $f_{2}$, respectively. (For interpretation of the references to colour in this figure legend, the reader is referred to the web version of this article.)

of ACHM traces. However, another deduction is also possible, namely that the higher frequency components, detected by the FFT, distinguish between the two conditions. This can be based on the observation that the main frequency peak in normal subjects was at
$140 \mathrm{~Hz}$, whereas in ACHM patients was at $100 \mathrm{~Hz}$, a significant difference. Furthermore, since the signal has been analysed up $50 \mathrm{~ms}$, the high frequency components could originate from part of the ascending limb of the $b$-wave. 
To exclude this possibility, we have repeated the FFT, by limiting the analysed ERG signal only to the first 25 ms. However, in this case, due to the smaller acquisition time, the new resolution of FFT worsens, making impossible the detection of differences in the frequency components. In particular, in $100 \%$ of the healthy traces we found the high frequency component at $160 \mathrm{~Hz}$, present also in the $50 \%$ of the ACHM ERGs.

With the purpose of further exploring the efficiency of FA we have carried out another study by enlarging the acquisition time up to $500 \mathrm{~ms}$ (in order to improve the frequency resolution), but investigating only the negative potential up to the cross-over with the time axis and leading to zero the remaining part of ERG signal. This data manipulation, clearly, introduces artifacts, but it limits the influence of the ascending limb of the $b$-wave. Figure 6 displays the outcomes of this investigation, showing the revealed frequency components for both healthy (full circles) and ACHM (empty circles) traces together with their occurrence. Due to the significant value of their occurrence, in the normal set it is possible to identify two more "characterizing" frequencies, namely 155 and $222 \mathrm{~Hz}$. On the other hand, the spread of detected frequencies does not provide information statistically relevant for the ACHM sample.

Our findings prove that the FA is not capable to discriminate, with a satisfactory degree of confidence, between ACHM and normal traces.

\section{Wavelet analysis}

Figure 7 shows the outcome of the WA performed on three waveforms, each of them belonging to a different set under investigation. In particular, panels of Fig. 7 show the spectral behaviour of a record representative of a normal subject, an ACHM and a CSNB patient, under the same luminance conditions as in Fig. 1. The three panels report the amplitudes of the wavelet transform in the 10$240 \mathrm{~Hz}$ range across time as colour contour plots, in which the correlation magnitudes are expressed in a pink scale. Each scalogram yields a dominant maximum (white cluster) $f_{0}$ and one or two local maxima, $f_{1}$ and $f_{2}$. The analysis of the clusters in the scalograms shows that it is possible to individuate in normal subjects three stable frequency components, related to the activity of the photoreceptors, whereas the values and/or the number of these frequencies are indicative of an eventual pathology [38,39,49].

The lower frequency $f_{0}$ is associated with the combined activities of the photoreceptors, in the sense that its value takes into account the temporal distribution of the amount of excited photoreceptors $[38,39]$. The higher frequencies $f_{1}$ and $f_{2}$ are related to the contributions of the photoreceptors ( $f_{1}$ to rods, $f_{2}$ to cones) and to their peculiarities [27]. These frequencies, in fact, show behaviours coherent with the features of the three set of traces. In the upper panel of Fig. 7, for example, they distinctly appear according with the normal response of both photoreceptors, whereas in the others panels one or two frequency components disappear consistently with the pathological photoreceptoral response. Their presence and value are, hence, indicative of the functional integrity of the retina. To optimize the characterisation of the $a$-waves, taking into account the behaviour differences concerning the consistency of the dynamics across healthy and pathological subjects, both the mean values of the frequencies $f_{0}, f_{1}$ and $f_{2}$ and their times of occurrence were analysed.

Figure 8 reports, for the investigated samples (healthy, ACHM and CSNB traces), the mean values of the frequencies appearing in each cluster of all scalograms and their times of occurrence. The timefrequency features detected by the WA allow us to classify with a high degree of confidence the belonging of each trace to a particular group. On the other hand, a separate analysis of the frequencies and/ or the occurrence times is unable to achieve this task since it is unable to discriminate contiguous frequencies falling within the

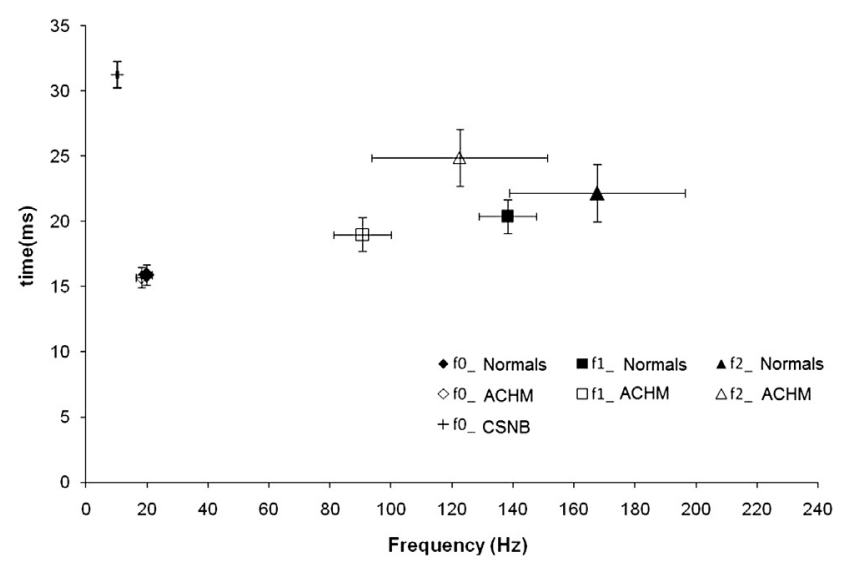

Figure 8. Mean values of the frequencies appearing in each cluster of all scalograms and their times of occurrence. Diamond, triangles and squares symbols refer to $f_{0}, f_{1}$ and $f_{2}$ frequencies, respectively.

standard deviation. This occurs in Fig. 8, for example, for the frequencies labelled $f_{1}$ and $f_{2}$ for the healthy subjects and $f_{2 \_ \text {ACHM }}$, or for $f_{1}$ and $f_{2}$ in ACHM patients. The same happens in the separate analysis of occurrence times. Otherwise, the joint analysis of both frequencies and occurrence times, not possible with conventional spectral techniques, such as FA, overcomes this inconvenience and provides significant information to classify the subjects.

WA findings are summarised in Table 2, that furnishes the most relevant statistical parameters regarding normal, ACHM and CSNB traces. It reports for each stable component, the mean value of frequency and occurrence time, their standard deviations (SD) the coefficients of variation (CV) and the appearance i.e. in how many subjects a certain frequency group can be identified. The values of $\mathrm{CV}$, defined according to $\mathrm{CV}=100 \times \mathrm{SD} /$ mean, allow us to estimate and compare the variability between different subjects within the same sample [39]. A comparison between the data shown in Fig. 7 and in Table 2, indicates that the sets of normal and ACHM subjects reveal minor changes in $f_{0}$ and significant differences in $f_{1}$ and $f_{2}$, whereas the CSNB patients present an appreciable difference also in $f_{0}$.

In ACHM traces, both frequencies $f_{1}$ and $f_{2}$ are present but with lower values than those of normal traces. This can be explained by the fact that, in this disease the rods are working properly, but their activity is affected by the abnormal response of the cones. The shift toward lower values of one frequency component can be linked with the pathological response of one photoreceptoral population. In fact, the lowering of the frequency values is associated with the anomalous activity of the cones that affects the crosstalk between them and the rods. This circumstance prevents the occurrence of correlation phenomena that characterize the healthy response of the photoreceptors. This assessment validates the current

Table 2

Values of the frequency components, times of occurrence, coefficients of variability, appearances extracted from the three samples of traces for luminance value log (I) $\left.I_{0}\right)=1.5$.

\begin{tabular}{lllll}
\hline $\log \left(I / I_{0}\right)=1.5$ & & & \\
\hline Sample & Freq. $(\mathrm{Hz}) \pm \mathrm{SD}$ & Time $(\mathrm{ms}) \pm \mathrm{SD}$ & $\mathrm{CV}($ Freq $) \%$ & Appearance $(\%)$ \\
\hline Normals & $f_{0}: 19.8 \pm 1.4$ & $t_{0}: 15.9 \pm 1.0$ & 7.3 & 100 \\
& $f_{1}: 138.2 \pm 6.9$ & $t_{1}: 20.4 \pm 2.8$ & 5.0 & 70 \\
& $f_{2}: 167.6 \pm 11.4$ & $t_{2}: 22.2 \pm 3.3$ & 6.8 & 90 \\
ACHM & $f_{0}: 18.1 \pm 1.8$ & $t_{0}: 15.7 \pm 0.8$ & 10.1 & 100 \\
& $f_{1}: 90.6 \pm 9.4$ & $t_{1}: 19.0 \pm 1.3$ & 10.35 & 50 \\
& $f_{2}: 122.5 \pm 28.8$ & $t_{2}: 24.9 \pm 2.2$ & 23.5 & 62.5 \\
CSNB & $f_{0}: 10.2235 \pm 0.205$ & $t_{0}: 31.25 \pm 1.1$ & 2.003 & 100 \\
& $f_{1}:-$ & $t_{1}:-$ & - & - \\
& $f_{2}:-$ & $t_{2}:-$ & - & - \\
\hline
\end{tabular}


knowledge about rod-cone interactions during the response in normal subjects and provides useful information about the frequency-temporal features of the ACHM pathology and its clinical interpretation. The differentiations in the pathological sample can be explained as follows: the contribution of the cones (reduced in number and not properly working) decreases in contrast with rods that maintain a "normal" behaviour.

On the other hand, the other investigated retinal pathology, represented by the CSNB $a$-waves, leads to the lacking of both frequency components. In CSNB, $f_{1}$ is absent because it is related to rod activity whereas $f_{2}$, bound to cones, does not appear since is necessary a higher luminance value for switching it. Moreover, WA analytical features resulting by adaptability of the width of the scale to the signal being analysed, allow a better resolution in $f_{0}$, identifying its value to about $10 \mathrm{~Hz}$.

\section{Conclusions}

The present paper applies for the first time three signal processing techniques, already used in the analysis of other biomedical temporal series, to the recognition of ERG waveforms. To achieve this task, ERG signals, recorded accordingly to routine clinical protocol are sufficient. Additional ad-hoc records, such as high intensity ERGs, that need an increase of both time and costs, are not required. In this context, we monitor the efficiency of these non standard techniques in discriminating and recognizing different photoreceptor diseases, even before the clinical diagnosis of specialists. These processing methods should make easier the work of medical doctors and show trends that are not obvious when performing a visual inspection of the recorded signal.

In our case, the simple analysis of both slope and implicit time of $a$-waveforms is successful in classifying CSNB ERGs, but does not provide enough information helpful from the clinical viewpoint, because there is not a statistically significant difference between the extrapolated mean values of ACHM and normal traces. Furthermore, also the use of well consolidated and widely used analytical methods, such as PCA and FA, is not able to furnish, with an high degree of confidence, for both the investigated pathologies, comprehensive information about the eventual diagnosis of the ERG records. In fact, both PCA and FA are successful in detecting the CSNB pathological traces, but provide controversial findings in discriminating the ACHM $a$-waves from the normal ones. In particular, the FA is strongly affected by the temporal interval of analysis, which is bound to the frequency resolution. This means that if we limit the FA of $a$-waves to the first $25 \mathrm{~ms}$, since the sampling time of traces has been fixed by the physician, contiguous frequencies are not distinct and even are then convoluted. This drawback, in the context of this investigation, can lead to an incorrect classification of the traces.

On the other hand, time-frequency analysis (WA) of ERGs yields wave peculiarities with good accuracy. This improvement is more evident in the recognition of waveforms in which small differences are not appreciable by a "naked eye" analysis. Thanks to the simultaneous assessment of both the frequency components and their time of occurrence, separation between normal and pathological waveforms is achieved, and consequently, WA plays a relevant role in clinical investigations. In fact, the WA utilization as a classification algorithm of ERGs, recorded according to the standard clinical protocol, allows us to improve and speed up the clinical diagnosis of certain photoreceptoral diseases. Moreover, the detection and distribution of the frequency components in the time-frequency frame, obtained through the WA, is the first step toward the development of an electroretinographic signal classifier, capable of recognizing different photoreceptoral pathologies helpful in the early detection of retinal diseases.
The analytical approach provided by the WA, although validated by results, needs further updates, both in terms of methodology and increasing of the statistical sample in order to carry out additional checks. This task is hampered by the infrequency of these pathologies.

\section{Acknowledgements}

The authors are very thankful to Prof. L. Bellomonte for the valuable help in data analysis and precious comments and thank Prof. M. Anastasi for providing the human ERG data. The authors would also to thank the anonymous reviewers who made substantive suggestions for improving this paper.

\section{References}

[1] Pal S, Mitra M. Detection of ECG characteristic points using multiresolution wavelet analysis based selective coefficient method. Measurement 2010;43. 255-61.

[2] Saritha C, Sukanya V, Narasimha Murthy Y. ECG signal analysis using wavelet transforms. Bulg J Phys 2008;35:68-77.

[3] Hu X, Yu Q, Liu W, Qin J, Feature extraction of surface EMG signal based on wavelet coefficient entropy, IEEE Xplore, 978-1-4244-1748-3/08.

[4] Kilby J, Gholam Hosseini H. Extracting effective features of SEMG using continuous wavelet transform. In: Proceedings of the 28th IEEE EMBS annual international conference New York City, USA; Aug 30-Sept 3, 2006.

[5] Burns JW, Consens FB, Little RJ, Angell KJ, Gilman S, Chervin RD. EMG variance during polysomnography as an assessment for REM sleep behavior disorder. Sleep 2007;30(12):1771-8.

[6] Schlögl A, Kemp B, Penzel T, Kunz D, Himanen SL, Värri A, et al. Quality control of polysomnographic sleep data by histogram and entropy analysis. Clin Neurophysiol 1999;110:2165-70.

[7] Granit R. The components of the retinal action potential in mammals and their relation to the discharge in the optic nerve. J Physiol 1933;77:207-39.

[8] Hood DC, Birch DG. A quantitative measure of the electrical activity of human rod photoreceptors using electroretinography. Vis Neurosci 1990;5:379-87.

[9] Lamb TD, Pugh Jr EN. A quantitative account of the activation steps involved in phototransduction in amphibian photoreceptors. J Physiol 1992;449:719-58.

[10] Pugh Jr EN, Lamb TD. Amplification and kinetics of the activation steps in phototransduction. Biochim Biophys Act 1993;1141:111-49.

[11] Jamison JA, Bush RA, Lei B, Sieving PA. Characterization of the rod photoresponse isolated from the dark-adapted primate ERG. Vis Neurosci 2001;18: 445-55.

[12] Saszik SM, Robson JG, Frishman LJ. The scotopic threshold response of the dark adapted electroretinogram of the mouse. J Physiol 2002;543:899-916.

[13] Fulton AB, Hansen RM, Westall CA. Development of ERG responses: the ISCEV rod, maximal and cone responses in normal subjects. Doc Ophthalmol 2003;107(3):235-41.

[14] Hood DC, Birch DG. Principles and practice of clinical electrophysiology of vision. Cambridge, MA: MIT Press; 2006487.

[15] Hamilton R, Bees MA, Hamilton R, Bees MA, Chaplin CA, McCulloch DL. The luminance-response function of the human photopic electroretinogram: a mathematical model. Vis Res 2007;47(23):2968-72.

[16] Nilsson J, Wright T, Westall C. Rod a-wave analysis using high intensity flashes adds information on rod system function in $25 \%$ of clinical ERG recordings. Vis Res 2008;48:1920-5.

[17] Sugawara T, Sieving PA, Bush RA. Quantitative relationship of the scotopic and photopic ERG to photoreceptor cell loss in light damaged rats. Exp Eye Res 2000;70(5):693-705.

[18] Birch DG, Hood DC, Locke KG, Hoffman DR, Tzekov RT. Quantitative electroretinogram measures of phototransduction in cone and rod photoreceptors: normal aging, progression with disease, and test-retest variability. Arch Ophthalmol 2002;120(8):1045-51.

[19] Usui T, Tanimoto N, Ueki S, Takagi M, Hasegawa S, Abe H, et al. ERG rod awave in Oguchi disease. Vis Res 2004;44(5):535-40.

[20] Roman AJ, Schwartz SB, Aleman TS, Cideciyan AV, Chico JD, Windsor EA, et al. Quantifying rod photoreceptor-mediated vision in retinal degenerations: dark-adapted thresholds as outcome measures. Exp Eye Res 2005;80:259-72.

[21] Sandmeyer LS, Grahn BH, Breaux CB. Diagnostic ophthalmology. Congenital stationary night blindness (CSNB). Can Vet J 2006;47:1131-3.

[22] Cideciyan AV, Jacobson SG. An alternative phototransduction model for human rod and cone ERG a-waves: normal parameters and variation with age. Vis Res 1996;36:2609.

[23] Pugh EN, Falsini B, Lyubarsky AL. The origin of the major rod-and cone-driven components of the rodent electroretinogram, and the effect of the age and light-rearing history on the magnitude of these components. In: Williams TP, Thistle AB, editors. Photostasis and related topics. New York: Plenum; 1998. p. 93-128.

[24] Burns E, Lamb TD. Visual transduction by rod and cone photoreceptors in Chalupa. In: L M, Werner JS, editors. The visual neurosciences 2003. p. 215-33. 
[25] Robson JG, Saszik SM, Ahmed J, Frishman L. Rod and cone contributions to the a-wave of the electroretinogram of the macaque. J Physiol 2003:509-30.

[26] Barraco R, Bellomonte L, Brai M, Anastasi M. Analysis of the human a-wave ERG component. Physiol Meas 2006;27:881-99.

[27] Barraco R, Persano Adorno D, Bellomonte L, Brai M. A study of the human rod and cone ERG a-wave component. J Stat Mechanics: Theory Exp 2009: P03007-11.

[28] Marmor F, Arden GB, Nilsson SEG, Zrenner E. International Standardisation Committee, standard for clinical electroretinography. Arch Ophthalmol 1989;107:816-9.

[29] Marmor F, Holder GE, Seeliger MW, Yamamoto S. Standard for clinical electroretinography (2004 update). Doc Ophthalmol 2004;108:107-14.

[30] Robson JG, Frishman L. Sampling and interpolation of the a-wave of the electroretinogram. Doc Ophthalmol 2004;108:171-9.

[31] Diamantaras KI, Kung SY. Principal component neural networks: theory and applications. New York: Wiley; 1996.

[32] Devijver A, Kittler J. Pattern recognition: a statistical approach. Englewood Cliffs, NJ: Prentice-Hall; 1989.

[33] Jain K. Fundamentals of digital image processing. Englewood Cliffs, NJ: Prentice-Hall; 1989.

[34] Stamkopoulos T, Diamantaras K, Maglaveras N, Strintzis M. ECG analysis using nonlinear PCA neural networks for ischemia detection. IEEE Trans Signal Process 1998;46(11):3058-67.

[35] Castells F, Laguna P, Sörnmo L, Bollmann A, Roig JM. Principal component analysis in ECG signal processing. EURASIP J Adv Signal Process, http://dx.doi. org/10.1155/2007/74580.

[36] Khakpoor F, Ardeshir G. Using PCA and SVD to improve wavelet-based method for detection of voice and silence in speech. Eur J Scientific Res 2009;37(4):641-8.

[37] Varadharajan S, Fitzgerald K, Lakshminarayanan V. A novel method for separating the components of the clinical electroretinogram. J Mod Opt 2007;54:1263-80.
[38] Barraco R, Persano Adorno D, Brai M. ERG signal analysis using wavelet transform. Theory in Biosciences 2011;130(3):155-63.. http://dx.doi.org/ 10.1007/s12064-011-0124-1.

[39] Barraco R, Persano Adorno D, Brai M. An approach based on wavelet analysis for feature extraction in the a-wave of the electroretinogram. Comput Methods Programs Biomed 2011;104:316-24.. http://dx.doi.org/10.1016/ j.cmpb.2011.05.001.

[40] Yinhong Z, Quanlu L, Jing W. The study of time-frequency analysis the nocturnal snoring signal based on the wavelet transform. J Acoust Soc Am 2012;131(4):3443.

[41] Ergen B, Tatar Y, Gulcur HO. Time-frequency analysis of phonocardiogram signals using wavelet transform: a comparative study. Comput Methods Biomech Biomed Eng 2012;15(4):371-81.

[42] Yuan Q, Zhou W, Zhang J, Li S, Cai D, Zeng Y. EEG classification approach based on the extreme learning machine and wavelet transform. Clin EEG Neurosci 2012;43(2):127-32.

[43] Rioul O, Vetterli M. Wavelet and signal processing. IEEE SP Magazine 1991:14-38

[44] Astafeva NM. Wavelet analysis: basic theory and some applications. PhysicsUspekhi Fizicheskikih Nauk 1996;39:1085-108.

[45] Forte JD, Bui BV, Vingrys AJ. Wavelet analysis reveals dynamics of rat oscillatory potential. J Neurosci Methods 2008;169:191-200.

[46] Durka PJ. From wavelets to adaptive approximations: time frequency parametrization of EEG. Biomed Eng Online 2003;2(1).

[47] Jedrzejczak WW, Blinowska KJ, Konopka W. Time-frequency analysis of transiently evoked otoacoustic emissions of subjects exposed to noise. Hear Res 2005;205:249-55.

[48] Marcus M, Cabael L, Marmor MF. Utility in clinical practice of standard vs. High-Intensity ERG A-Waves, Documenta Ophthalmologica 2006;113: 145-53.

[49] Miguel-Jimenez JM, Ortega S, Boquete L, Rodriguez-Ascariz JM, Blanco R Multifocal ERG wavelet packet decomposition applied to glaucoma diagnosis. BioMedical Eng OnLine 2011:10-37. 